\title{
Rehabilitative treatment of cleft lip and palate: experience of the Hospital for Rehabilitation of Craniofacial Anomalies - USP (HRAC-USP) - Part 2: Pediatric Dentistry and Orthodontics
}

\author{
José Alberto de Souza FREITAS ${ }^{1}$, Daniela Gamba GARIB ${ }^{2}$, Thais Marchini OLIVEIRA ${ }^{3}$, Rita de Cássia Moura Carvalho \\ LAURIS $^{4}$, Ana Lúcia Pompéia Fraga de ALMEIDA ${ }^{5}$, Lucimara Teixeira NEVES ${ }^{6}$, Ivy Kiemle TRINDADE-SUEDAM ${ }^{6}$, \\ Renato Yassutaka Faria YAEDÚ7, Simone SOARES ${ }^{5}$, João Henrique Nogueira PINTO
}

\begin{abstract}
1- DDS, MSc, PhD, Superintendent, Hospital for Rehabilitation of Craniofacial Anomalies and Full Professor, Department of Stomatology, Bauru School of Dentistry, University of São Paulo, Bauru, SP, Brazil.

2- DDS, MSc, PhD, Associate Professor of Orthodontics, Department of Pediatric Dentistry, Orthodontics and Community Health, Bauru School of Dentistry and Hospital for Rehabilitation of Craniofacial Anomalies, University of São Paulo, Bauru, SP, Brazil.

3- DDS, MSc, PhD, Assistant Professor, Department of Pediatric Dentistry, Orthodontics and Community Health, Bauru School of Dentistry and Hospital for Rehabilitation of Craniofacial Anomalies, University of São Paulo, Bauru, SP, Brazil.

4- DDS, MSc, Orthodontist of the Dental Division of the Hospital for Rehabilitation of Craniofacial Anomalies, University of São Paulo, Bauru, SP, Brazil.

5- DDS, MSc, PhD, Assistant Professor, Department of Prosthodontics, Bauru School of Dentistry and Hospital for Rehabilitation of Craniofacial Anomalies, University of São Paulo, Bauru, SP, Brazil.

6- DDS, MSc, PhD, Assistant Professor, Department of Biological Sciences, Bauru School of Dentistry and Hospital for Rehabilitation of Craniofacial Anomalies, University of São Paulo, Bauru, SP, Brazil.

7- DDS, MSc, PhD, Assistant Professor, Department of Stomatology, Bauru School of Dentistry and Hospital for Rehabilitation of Craniofacial Anomalies, University of São Paulo, Bauru, SP, Brazil.

8- DDS, MSc, PhD, Prosthodontist of the Dental Division of the Hospital for Rehabilitation of Craniofacial Anomalies, University of São Paulo, Bauru, SP, Brazil.
\end{abstract}

Corresponding address: Daniela Gamba Garib - Faculdade de Odontologia de Bauru - USP - Alameda Dr. Octávio Pinheiro Brisolla, 9-75 - Bauru, São Paulo - 17012-901 - Brazil - Phone: 551432358282 - Fax: 551432234679 - e-mail: dgarib@uol.com.br

Received: December 16, 2011 - Accepted: April, 11, 2012

\section{ABSTRACT}

The aim of this article is to present the pediatric dentistry and orthodontic treatment protocol of rehabilitation of cleft lip and palate patients performed at the Hospital for Rehabilitation of Craniofacial Anomalies - University of São Paulo (HRAC-USP). Pediatric dentistry provides oral health information and should be able to follow the child with cleft lip and palate since the first months of life until establishment of the mixed dentition, craniofacial growth and dentition development. Orthodontic intervention starts in the mixed dentition, at 8-9 years of age, for preparing the maxillary arch for secondary bone graft procedure (SBGP). At this stage, rapid maxillary expansion is performed and a fixed palatal retainer is delivered before SBGP. When the permanent dentition is completed, comprehensive orthodontic treatment is initiated aiming tooth alignment and space closure. Maxillary permanent canines are commonly moved mesially in order to substitute absent maxillary lateral incisors. Patients with complete cleft lip and palate and poor midface growth will require orthognatic surgery for reaching adequate anteroposterior interarch relationship and good facial esthetics.

Key words: Cleft lip. Cleft palate. Pediatric dentistry. Orthodontics.

\section{INTRODUCTION}

Cleft lip and palate are the most prevalent malformations in mankind and are considered a relevant public health problem by the World Health Organization $^{47}$. In Brazil 5,800 births are registered per year ${ }^{47}$, with a prevalence of 1 in every 650 births ${ }^{46}$. The clefts may affect the lip, alveolar ridge and palate and cause esthetic, functional and psychosocial disorders. They occur in the embryonic period of intrauterine life and present multifactorial etiology, with association of genetic and environmental factors ${ }^{15,28}$.

The treatment of cleft lip and palate should be initiated soon after birth and continues up to adulthood, requiring the participation of 
an interdisciplinary team. The morphological rehabilitation of clefts involves plastic lip surgery at 3 months of age and palate surgery around 1 year of age, as well as secondary alveolar bone graft performed between 9 and 12 years of age ${ }^{46}$. Besides the primary plastic surgeries, the rehabilitation requires an interdisciplinary protocol involving different specialties as speech therapy, maxillofacial surgery and oral rehabilitation and the therapeutic procedures should be standardized 46 .

In dental rehabilitation, the pediatric dentistry provides oral health information and should be able to follow the child with cleft lip and palate since the first months of life until establishment of the mixed dentition, craniofacial growth and dentition development. The orthodontist monitors the craniofacial growth and development and corrects the malocclusions, which are more complex compared to patients without clefts. A great part of individuals present marked skeletal discrepancies in anteroposterior, transverse and vertical directions. This evidences the fundamental role of the maxillofacial surgeon working together with the orthodontist. This paper describes the treatment protocol of pediatric dentistry and orthodontic in individuals with cleft lip and palate.

\section{1 - PEDIATRIC DENTISTRY}

The presence of cleft lip and palate in infants often causes alterations in the feeding process. Breastfeeding for children with clefts is one of the most questioned aspects and raises doubts among caretakers. According to a previous study ${ }^{39}$, few children with cleft are breastfed and many mothers believe that can never breastfeed their children with cleft lip and palate. The mother may experience some difficulties depending on the type and extent of the cleft, yet breastfeeding should always be encouraged, because the child and mother may find mechanisms to adapt to the presence of the cleft and allow the breastfeeding, which is important for the child in the first months of life $e^{5,39}$.

The more complex the cleft, the greatest is the difficulty for the child to extract the milk directly from the breast. Though present, the swallowing reflex may be ineffective because of the deficient intraoral negative pressure caused by the cleft palate. In case of clefts affecting the lip, the difficulty is related to the inadequate lip sealing to grasp the breast, reducing the pressure applied on the nipple; in this situation, the mother may attempt to seal the defect with the breast. In clefts affecting the palate the child is unable to apply negative pressure to extract the milk; in these cases, breastfeeding will depend on the mother's attempt and the infant's adaptation ${ }^{5}$.

When breastfeeding is not possible, the mother is instructed to feed the baby using a bottle with latex nipple. Silicone nipples are not recommended because they are more rigid and may cause ulcerated lesions at the cleft area. The nipple should have orthodontic shape with a 0.8 to $1 \mathrm{~mm}$ orifice, so that the milk may drop and the child must suck strongly to extract it, exercising the orofacial musculature. Besides the bottle, other options for feeding include the use of disposable cups or spoons.

Both in natural and artificial feeding, the mother should be instructed that the infant should be in vertical position during feeding (half-seated in an approximate inclination of 45 degrees), reducing the risk of choking and milk reflux to the nose and auditory tube, which is horizontal at this age. The tube dysfunction and communication between oral and nasal cavities because of the cleft palate make these children more susceptible to recurrent ear infections, which may interfere with the audition in the long term ${ }^{5}$. The deficient filtering and heating of the inspired air and the difficult humidification predispose to airway infections, causing common colds, rhinopharyngitis and pharyngotonsillitis up to bronchitis and bronchopneumonia. The cleft palate also predisposes to the reflux of food and secretions, which favors the aspiration to the auditory tube and the occurrence of otitis media. These infections often relapse and, notwithstanding the adequate treatment, they may lead to hypoacusia (diminished auditory sensitivity) by accumulation of secretion in the middle ear and conductive hearing loss.

Other important aspect concerning the use of baby bottles concerns the early introduction of sugar in the diet. Sugar is usually used in the diet for weight gain, to allow the accomplishment of repair surgeries, and also for cultural reasons. The first contact with sugar should ideally be delayed as much as possible, so that the child might incorporate a healthy dietary habit ${ }^{39}$.

According to the literature, individuals with cleft lip and palate may present alterations in the deciduous dentition at the cleft area, especially affecting the maxillary lateral incisor ${ }^{16,25}$. The oral cavity of the newborn may present gingival and palatal cysts of the newborn, natal and neonatal teeth at the region of complete unilateral and bilateral cleft lip and palate, which may be lateral incisors of the normal series or supernumerary teeth ${ }^{1,25}$. These teeth present superficial implantation and excessive mobility, thus their extraction is indicated because of the risk of aspiration, due to the communication between the oral and nasal cavities in this type of cleft? .

Development of the deciduous dentition in individuals with cleft lip and palate presents delayed eruption of teeth at the cleft side. Tooth eruption may be delayed up to two years in complete 
cleft lip and palate. The deciduous dentition may present alterations proportional to the extent of the cleft, with greater involvement in more extensive clefts, except for isolated cleft palate, in which the alveolar ridge integrity is maintained. Dental anomalies of shape, structure, number and position are present mainly in teeth close to the cleft ${ }^{16,18,23}$. All these anomalies in the deciduous dentition may predispose the affected teeth to greater accumulation of dental biofilm and consequently to dental caries. Thus, counseling and follow-up are important to maintain the integrity of teeth, even supernumerary or malpositioned teeth, in order to maintain the supporting bone structures, which may be defective at the cleft area11,14,19.

The mixed dentition presents specific characteristics in the different types of clefts. The literature reports an overall delay of 6 months in the formation of permanent teeth in children with complete cleft lip and palate $e^{9,14,19,37}$. There is also high prevalence of ectopic eruption of the permanent maxillary first molar, which is related to the smaller anteroposterior length and retropositioning of the maxilla in relation to the cranial base in individuals with clefts affecting the palate ${ }^{9,14,19,37}$.

The literature has demonstrated that enamel structure alterations are frequent in permanent central incisors adjacent to the alveolar cleft ${ }^{23,45}$. When permanent lateral incisors are present at the cleft region, they present high prevalence of shape alterations, with peg-shaped aspect. In the permanent dentition the lateral incisor may be missing in 20 to $26 \%$ of cases of unilateral cleft lip and in $50 \%$ of complete unilateral cleft lip and palate $23,32,45$.

The rehabilitation of individuals with cleft lip and palate is directly related to the oral condition, which is a basic requirement for the accomplishment of repair surgeries, which require an infection-free environment for a satisfactory outcome. The oral hygiene care should be initiated before eruption of the first teeth. Cleaning of the soft tissues in this stage should be performed with gauze or cloth moistened with filtered or boiled water around the caretaker's forefinger, at least once a day, to create a healthy oral environment and contribute to the early acquisition of oral hygiene habits. After eruption of the first teeth, toothbrushing should be performed with a small quantity of toothpaste, which is an adequate method to prevent oral diseases as dental caries and gingivitis, which may damage the rehabilitative process. Fluoridated dentifrices should be carefully used to avoid the risk of fluorosis due to excessive ingestion of dentifrice. Special attention should be paid to teeth in ectopic position at the cleft area, in which the access for toothbrushing is complicated.

Diet may be a risk factor to dental caries and the parents should receive proper counseling. Other risk factors are related to the malformation, such as supernumerary and malpositioned teeth or teeth with shape or structure alterations, while others are related to the repair surgeries, such as lip fibrosis and presence of bridles. The pediatric dentist must educate, motivate and inform the parents on the need to maintain the oral health of their children for the success of rehabilitation. The world literature is not unanimous concerning the higher prevalence of dental caries in children with cleft lip and palate compared to those without clefts ${ }^{6,11}$.

The emotional aspect should also be considered for the family of children with cleft lip and palate, which may give rise to negligent attitudes on oral hygiene, because of the fear to manipulate the child's oral cavity or even the attempt to avoid unpleasant procedures ${ }^{10}$. The pediatric dentist should prioritize the constant and adequate attention to oral hygiene of individuals with cleft lip and palate, by educational and preventive programs for dental plaque control.

The pediatric dental treatment for children with cleft lip and palate is not different from the conventional dental treatment concerning the prevention of dental caries and periodontal disease. The management techniques are those routinely used for child behavior control, considering the normal psychological development ${ }^{30}$. Clinical examination and an adequate restorative and preventive treatment plan, as well as parent counseling, is fundamental for the future rehabilitation of individuals with cleft lip and palate. However, the anatomical alterations brought about by the anomaly may cause differences that influence the dental treatment ${ }^{30}$.

Dental anesthesia in individuals with cleft is not different for most regions in the oral cavity, except for the cleft area. At this region, the maxilla is divided in different segments by the bone defect, with individual innervation. Even though the clinical aspect is improved after surgical repair, the alveolar separation is maintained ${ }^{38}$. This is important when teeth at this region must be anesthetized, because the malpositioning may complicate determination of the site of tooth implantation. Therefore, previous periapical radiographic examination is recommended to analyze the bone segment in which the tooth is implanted. The surgical lip repair usually causes a secondary scar fibrosis at the region, making the mucosa more resistant and consequently the puncture is more painful. The initial puncture with anesthetic infiltration should be parallel to the tooth long axis. Because of the bone defect that separates the innervation of the two cleft segments, the adjacent region must also be anesthetized to avoid pain or discomfort during treatment, using the same puncture as the 
initial infiltration, yet directing the needle to this region. Anesthesia of the palatal region is always necessary ${ }^{38}$.

Rubber dam isolation is recommended for dental treatment whenever possible, especially in cases of unrepaired cleft palate. The rubber dam isolates the constant water flow of the high speed handpiece, dental caries or restorative material remnants, avoiding their penetration in the airway, which communicates with the oral cavity in these individuals. The dental clasp should be carefully placed using dental floss ligatures to avoid the risk of aspiration. This also applies when dental clasps are placed in supernumerary, rotated or malpositioned teeth.

The presence of carious lesions leads to the need of restorative treatment, if detected on time, or tooth extraction if the extent of the lesion does not allow restoration. The atraumatic restorative technique should be indicated for initial carious lesions without risk of pulp contamination. Whenever individuals with cleft lip and palate present dental caries with risk of pulp contamination, treatment should be conventionally performed. Individuals submitted to surgery should have an excellent oral condition, removing the sources of infection that may compromise the surgery ${ }^{13}$. Supernumerary and/or malpositioned deciduous teeth adjacent to the cleft should be maintained as long as possible, in order to preserve bone tissue that is already defective at this region.

According to the documentation protocol of Hospital for Rehabilitation of Craniofacial Anomalies (HRAC/USP), impression and photographs are obtained at pre-established periods: before cheiloplasty - around 3 to 6 months of age; before palatoplasty - around 12 months of age; 1 year after the last primary surgery; and complete deciduous dentition. Before initiating the dental arch impressions for achievement of dental casts, the parents are informed on the need of this procedure, how it is performed and is reassured in case of pain and/or nausea. The patients were molded and photographed since first months (Figure $1 \mathrm{~A}-\mathrm{C}$ ). Before 4 years of age impressions are obtained only for the maxillary dental arch. Impressions of the mandibular arch are only obtained in the complete deciduous dentition.

Knowledge on these aspects is important for the rehabilitative treatment to be performed at this stage, which comprises the onset of orthodontic intervention and accomplishment of secondary surgeries, including alveolar bone graft. The pediatric dentist must also diagnose the malocclusions and refer for orthodontic treatment in adequate timing.

\section{2 - ORTHODONTICS}

The malocclusions observed in individuals with clefts present peculiar complexities that differentiate them from occlusal irregularities of individuals without clefts. In general, patients with complete cleft lip and palate have two intra-arch and two interach disorders, as follows:

Tooth malpositioning and dental anomalies

In clefts affecting the alveolar ridge a peculiar tooth malpositioning is commonly observed, represented by the presence of contra-angulated and rotated maxillary central incisor, with the crown turned toward the distal side filling part of the cleft space, while the apex is mesially displaced to avoid the bone defect. The maxillary canine also tends to present excessive mesial angulation, with the crown turned toward the defect and often determining a Class II sagittal relationship in the smaller segment, even in the presence of a Class III skeletal pattern. In unilateral clefts, the maxillary midline is usually deviated to the cleft side, leading to the need of asymmetric extraction in the maxillary arch in some cases, especially in the presence of crowding. Tooth crowding is a frequent characteristic in the maxilla especially because of the deficient sagittal and transversal maxillary growth.

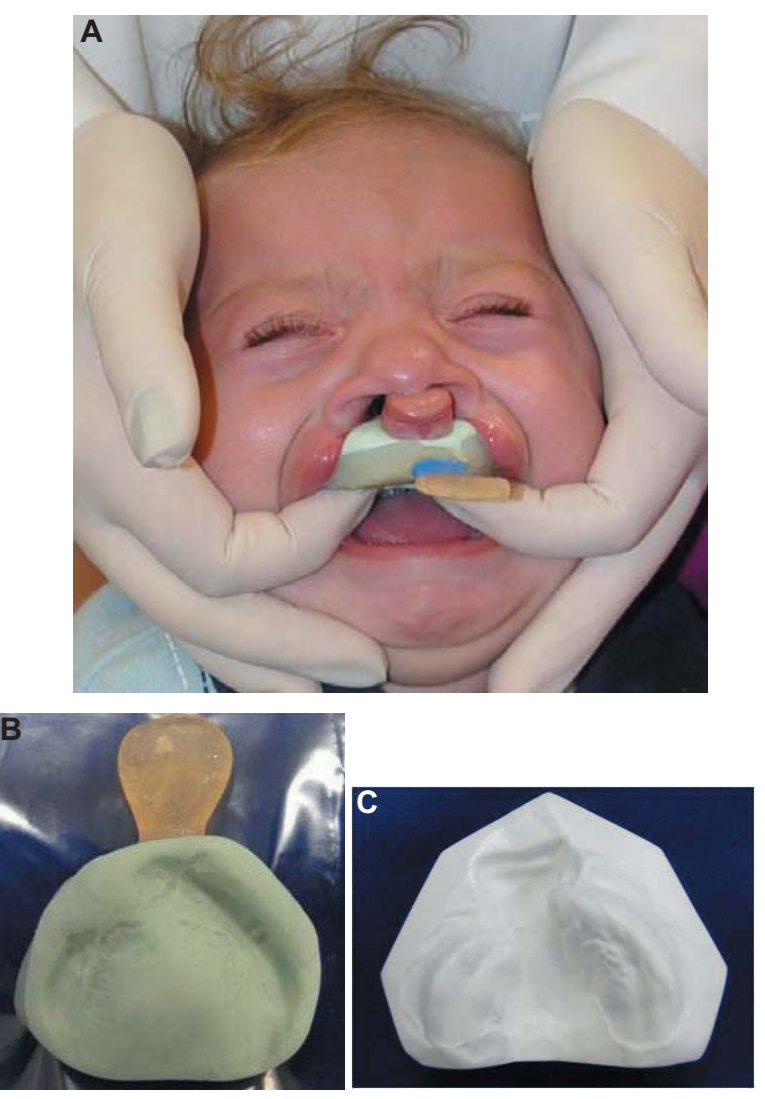

Figure 1 A-C- Impressions and plaster models of the maxillary dental arch for diagnosis and treatment plan (parents authorized the publication of this picture) 
Dental anomalies of number, shape and position (ectopic eruption) are also frequently observed in individuals with clefts $^{20,26,31}$. In the permanent dentition, the agenesis of maxillary lateral incisor is the most common anomaly, followed by the presence of a supernumerary lateral incisor located distal to the cleft ${ }^{31}$. Hypodontia of maxillary and mandibular second premolars and third molars is also frequent, with increase prevalence compared to the general population. Ectopic eruption of the maxillary first molar is observed in $20 \%$ of individuals with complete cleft lip and palate ${ }^{40}$.

\section{Bone defect at the anterior alveolar ridge}

Even though the primary plastic surgeries reconstruct the morphological lip and palate soft tissue defects in early childhood, the alveolar and palatal bone defects of individuals with clefts persist, hidden under the oral mucosa (Figure 2). The alveolar bone defect limits the possibility of tooth movement at this region because of the risk of dehiscences and fenestrations in teeth adjacent to the cleft. Previously, hypodontia of the maxillary lateral incisor frequently observed in individuals with clefts affecting the alveolar ridge could only be treated by conventional prosthetic rehabilitation. The orthodontic treatment usually comprised leveling of the maxillary arch with contra-angulation of the maxillary central incisor and over-angulation of the maxillary canine, both adjacent to the cleft. The enamel of these teeth was partially ground for prosthetic rehabilitation.

Introduction of the secondary bone graft procedure in the rehabilitation protocol widened the perspectives of corrective orthodontic treatment and overcame its limitations. After the publication of successful outcomes in the center of Oslo, Norway ${ }^{4}$,

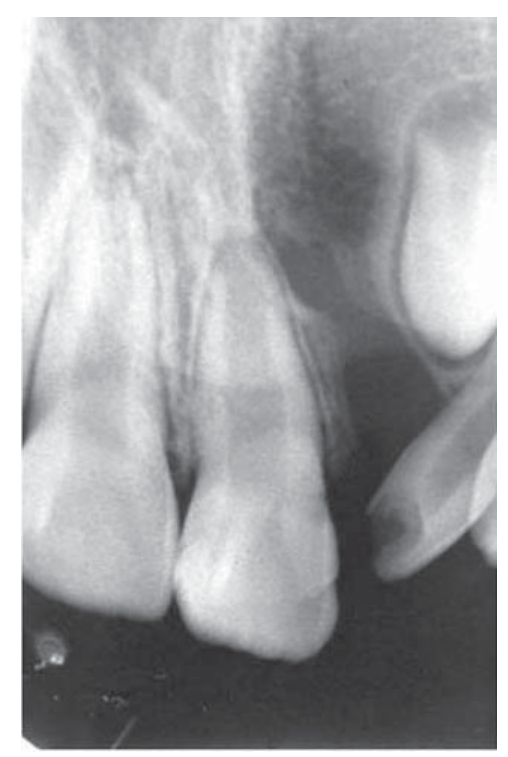

Figure 2- Image of the alveolar bone defect in the cleft region this procedure has been performed at HRAC-USP since the 1990 s with success rates of 80 to $90 \%$. The bone graft reconstructs the bone anatomy of the cleft alveolar ridge, allowing tooth movement at the region of maxillary lateral incisors.

\section{Sagittal maxillary deficiency}

Individuals with complete unilateral cleft lip and palate present a marked and progressive restriction of maxillary anteroposterior growth, essentially caused by primary plastic surgery. The tension on the reconstructed lip, as well as the scar caused by cheiloplasty, restricts the maxillary anterior growth $^{8}$. The early palatoplasty also seems to present a restrictive influence, though to a lesser extent than cheiloplasty, on sagittal maxillary growth $8,21,22$. The wider the cleft at birth, the less protruded is the maxilla in the mixed dentition ${ }^{12}$.

This restrictive effect of the maxillary growth ultimately causes a skeletal Class III pattern because of the maxillary deficiency ${ }^{34,36}$. Consequently, the anterior crossbite is a frequent occlusal characteristic in individuals with complete clefts operated in childhood. Individuals with complete bilateral cleft lip and palate present similar characteristics ${ }^{33}$. Individuals with cleft lip (affecting only the lip and alveolar ridge) and individuals with cleft palate (affecting only the palate) do not present deficiencies in anteroposterior maxillary growth after plastic surgeries ${ }^{44,46}$.

\section{Transverse deficiency of the maxillary dental arch}

The absence of a midpalatal suture leads to reduced transverse dimensions of the maxillary dental arch in individuals with complete cleft lip and palate compared to individuals without clefts ${ }^{41,43}$. The early palatoplasty increases this effect, causing further transverse maxillary deficiency ${ }^{41,43}$. Therefore, posterior crossbite is frequently observed in individuals with complete clefts, requiring expansion procedures during orthodontic intervention. Especially in the permanent dentition, individuals with clefts commonly present a natural compensation of the mandibular arch to the maxillary atresia, with excessive lingual tip of crowns of mandibular premolars and molars.

\section{1- ORTHODONTIC DIAGNOSIS}

The diagnosis of malocclusions in individuals with clefts makes use of the same resources employed in conventional orthodontic records: facial and dental photographs, dental casts, extraoral and intraoral radiographs. In general, at HRAC-USP, orthodontic records are taken at 9 years, 12 years and 18 years of age. 


\section{Facial analysis}

Since childhood, the facial analysis of patients with complete unilateral cleft lip and palate often reveals the deficient projection of zygomatic bone related to the deficient maxillary growth in sagittal direction. The Class III facial pattern is frequent with the upper lip positioned behind the lower lip. The marked anteroposterior maxillary deficiency may cause exposure of the eye sclera and aspect of exophthalmos. The mandible frequently presents normal or reduced size, with predominant characteristics of vertical growth, with small projection of the chin and short chin-neck line and closed chin-neck angle ${ }^{42}$. Even in the presence of marked maxillary deficiency the nasolabial angle is closed ( 85 to $90^{\circ}$ ) because of the low position of the nasal columella ${ }^{46}$. In frontal facial analysis, the deficient middle facial third may be apparent and the nose asymmetries are evident.

In complete bilateral cleft lip and palate, the facial analysis during childhood shows a convex profile, yet adolescents and adults present predominantly concave profiles, expressing the sagittal maxillary deficiency. Individuals with complete bilateral clefts present similar mandibles as individuals with complete unilateral clefts, with predominance of hyperdivergent growth ${ }^{42}$.

It should be highlighted that individual variations in facial pattern are frequent, yet the most characteristic patterns have been described. Also, it should be mentioned that individuals with incomplete clefts do not present sagittal deficiency of the middle facial third. The faces of individuals with clefts affecting only the lip and alveolar ridge are similar to the faces of individuals without clefts ${ }^{4}$. Conversely, individuals with cleft palate usually present maxillary and mandibular retrusion with hyperdivergent growth pattern ${ }^{44}$.

\section{Cephalometrics}

Individuals with operated complete unilateral cleft lip and palate present severe sagittal maxillary deficiency, with significant reduction of angles SNA and SN.ANS. A longitudinal study conducted at the center of Oslo-Norway ${ }^{34}$ demonstrated that the SNA angle is already reduced in early ages (79.6 at 5 years). During growth, the maxillary deficiency presented progressive reduction, reaching a mean SNA value of $74.2^{\circ}$ at 18 years of $\mathrm{age}^{34}$. The restriction at the ANS level is less expressive compared to point $A$. The mandible presents vertical growth morphology, with open gonial angle and downward and backward rotation of the mandibular plane ${ }^{42}$. The mandibular body is short compared to individuals without clefts ${ }^{42}$.

In individuals with complete bilateral cleft lip and palate, restriction of the maxillary anterior development is even greater, considering that the
SNA angle decreases $10^{\circ}$ from 5 to 18 years (from $84.9^{\circ}$ to $75^{\circ}$ ), yet the ANB angle is not as reduced as in individuals with complete unilateral cleft lip and palate, due to the initial protrusion of the premaxilla in these individuals ${ }^{34}$. The mandibular morphology of individuals with bilateral clefts is similar to individuals with complete unilateral clefts ${ }^{42}$.

The cephalometric pattern of individuals with cleft palate (isolated cleft palate) evidence short cranial base, retrusion of maxilla and mandible and vertical facial pattern ${ }^{44}$. Individuals with cleft lip present similar cephalometric characteristics as individuals without clefts ${ }^{46}$.

\section{Panoramic and intraoral radiographs}

The panoramic radiograph is used for a global view of the dentition and diagnosis of dental anomalies. The standardized occlusal radiograph of the cleft area, as well as the periapical radiograph of the cleft area, are often used for the assessment of the width and extent of the alveolar bone defect and for the follow-up of alveolar bone grafts.

\section{Dental casts}

Dental casts demonstrate the interarch relationship in sagittal, transverse and vertical directions, as well as intra-arch irregularities as tooth malpositioning and dental crowding. In patients with unilateral complete cleft lip and palate, Goslon Yardstick occlusal scores are used for defining the prognosis of the orthodontic treatment ${ }^{2,24}$. A new Yardstick was recently published for application in individuals with complete bilateral clefts ${ }^{29}$.

Dental casts evaluation is and important method for intercenter studies of treatment outcome 3,27. Occlusal scores are preferred in investigations because they present to be reproducible according to the Kappa ${ }^{35}$. In summary, the methods for evaluation of dental arches in individuals with clefts are much different from parameters employed in individuals without clefts in Orthodontics. Angle's classification is not as used as the Yardsticks in individuals with complete cleft lip and palate.

\section{2-ORTHODONTIC TREATMENT}

The orthodontic treatment of individuals with clefts follows these protocol stages:

1) Orthodontics before alveolar bone graft;

2) Secondary alveolar bone graft;

3) Orthodontics after alveolar bone graft;

4) Orthognathic surgery;

5) Finalization and retention;

\subsection{1- PRE-GRAFT ORTHODONTICS}

At our rehabilitation center, the orthodontic 
treatment is initiated in the middle-late mixed dentition, approximately at 8 years of age. This first intervention aims to correct the maxillary arch transversal deficiency and the posterior crossbite preparing the maxillary arch to receive the secondary alveolar bone graft. Rapid maxillary expansion with Hyrax or Haas type expanders is initiated when the permanent maxillary canines present formation of half to two thirds of the root (Figure $3 \mathrm{~A}$ ). The parameter of dental age is more important than the chronological parameter, due to the delayed dental development usually observed in individuals with clefts ${ }^{9}$. The maxillary arch transversal deficiency is not always associated with crossbite, especially in the presence of combined atresia of the mandibular dental arch. Even in these cases, there is the need of maxillary orthopedic expansion to prepare the maxilla to receive the secondary alveolar bone graft. Maxillary segments should be aligned to provide lateral walls for performing the alveolar bone graft.

After maxillary expansion, a fixed palatal arch retainer should be placed and maintained until postbone graft stage (Figure 3B). This fixed retainer is also maintained concomitantly with fixed appliances during the comprehensive orthodontic treatment.

Very early orthodontic interventions were discontinued at our center because of the high probability of relapse in the long term. Interventions at the deciduous and early mixed dentitions represent overtreatment, since it exhausts the individuals that are already submitted to a long and complex rehabilitation process, without expressive outcomes in the long term.

The anterior crossbite in individuals with occlusal
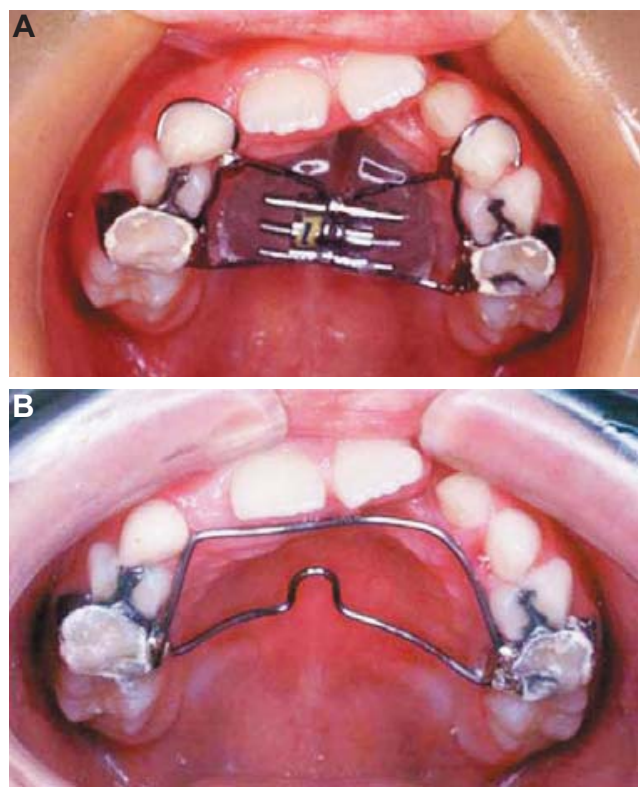

Figure 3- A- Haas type expander used for correcting the transversal deficiency of the maxillary arch. B- Fixed retainer used after rapid maxillary expansion score Goslon 3 may be corrected at this stage. When the anterior crossbite is skeletal due to the maxillary deficiency, the facial mask is used for maxillary protraction (MXP) after the maxillary expansion. On the other hand, in the presence of a dental crossbite, as observed in individuals with bilateral clefts presenting marked retroclination of the premaxilla and good facial esthetics, orthodontic appliances are used. For this purpose, modified quadri-helix appliance with anterior extensions or using leveling archwires with loops or omega loops associated to partial $4 \times 2$ fixed appliances are used. In individuals with complete bilateral cleft lip and palate treated with MXP, an anterior extension may be soldered in the Haas expander, adapted to the palatal surfaces of maxillary incisors, which may also move the premaxilla forward during the protraction.

When the individual presents posterior crossbite associated with anterior crossbite, such as individuals with oclusal score Goslon 3, the treatment planning involves expansion followed by anterior crossbite correction. However, in individuals with complete bilateral clefts, where the collapse of posterior segments with the premaxilla may superimpose the lateral incisors over the canines, the anterior crossbite is corrected before the posterior crossbite. Pre-bone graft orthodontic interventions may expose oronasal fistulae at the alveolar region, which will be opportunely closed during bone graft surgery, reducing the need of another plastic surgery.

Also, the orthodontist should be aware that the thin periodontal bone surrounding the teeth next to the alveolar cleft constitutes a limitation for tooth movement previously to alveolar bone graft procedure in patients with clefts ${ }^{16}$. Four important aspects should be considered during orthodontic treatment before bone graft:

1) Rotated teeth adjacent to the cleft should not be corrected before the bone graft surgery, because of the risk of dehiscences and fenestrations;

2) Supernumerary teeth erupted in the palatal side of clefts should be extracted at least three months before the bone graft, because the palatal mucosa may not be interrupted to cover the entire graft;

3) Orthodontic treatment planning before bone graft should always be performed in combination with the maxillofacial surgeon, in order to maximize the success of the future graft. For example, if the maxillary canine eventually erupts before the bone graft surgery, it may present infraversion because of the anatomical limitation at the cleft area. In this case, if the canine is leveled before the secondary bone graft, usually with extrusive and distal movement, the bone level of the alveolar crest after bone graft will be more favorable. Individual 
movement of the canine may be performed using a TMA wire cantilevers anchored to the molar. This should be anchored bilaterally with a transpalatal bar to avoid the collateral movement effect;

4) The orthodontist should always encourage patients on the need of adequate oral hygiene at this stage, since the success rate of bone grafts is lower in the presence of gingivitis.

\subsection{2- POST-GRAFT ORTHODONTICS}

In addition to the clinical evaluation, periapical and occlusal radiographic examination of the graft area allow qualitative and quantitative evaluation of the grafted bone. At this stage, if the canines are not yet erupted, the orthodontist should only follow the development and eruption of canines through the graft. If the canines are already erupted, corrective orthodontic treatment may be initiated 60 to 90 days after SABG.

Comprehensive orthodontic treatment of patients without or with mild midfacial deficiency is initiated when the permanent dentition is completed (Figure 4 and 5). Conversely, the post-graft orthodontic intervention should be postponed to age 16-17 years in patients with moderate to severe maxillary sagittal deficiency who need orthognatic surgery for maxillary advance. The planning for the comprehensive orthodontic treatment should include intra-arch and inter-arch objectives. The intra-arch therapeutic options include:

1) Maintenance of the maxillary lateral incisor in the cleft area when it is present and show a good root length;

2) Space closure of the region of missing maxillary lateral incisor by orthodontic mesialization of posterior teeth at the cleft side;

3) Space maintenance of the missing lateral incisor for implant placement after completion of orthodontic treatment;

4) Implant placement at the canine-premolar region after moving the maxillary canine toward mesial at the space of the absent lateral incisor. The advantage of this option compared to the previous one would be the prevention of bone loss at the SABG area.

The decision between space closure or maintenance should consider three main factors: position in which the maxillary canine erupted, tooth-size discrepancy, and sagittal relationship between the dental arches. The most favorable situation for space closure is when the canine erupts mesially, in the presence of maxillary tooth crowding and Class II sagittal relationship between dental arches at the cleft side. The clinical condition in which the canine erupts in habitual positioning, distal to the cleft, with Class I sagittal relationship between dental arches, and null or positive tooth-size discrepancy in the maxillary arch may be eligible for finalization using implants/ dental prostheses. Patient and family opinion is also important in making the decision. If mandibular tooth crowding is present, extraction of mandibular teeth may be planned associated to space closure in the maxillary arch.

In cases of unilateral clefts, deviation of the maxillary midline and crowding in the greater segment may determine the need of asymmetric extractions in the maxilla. In this case, it is recommended to extract one premolar or the lateral incisor, at the side opposite to the cleft. The maxillary lateral incisor may be eligible for extraction in cases with agenesis of the lateral incisor adjacent to the cleft, in order to achieve symmetry and smile esthetics at the end of treatment.

There are only two inter-arch therapeutic options for patients with Class III skeletal pattern. The selection will depend on the initial occlusal index as well as on the facial esthetics.

1) Compensatory orthodontic treatment: indicated for individuals with occlusal scores Goslon 3 and acceptable facial esthetics, since the orthodontic treatment alone does not improve the facial appearance. Mandibular extractions may be necessary in this therapeutic option.

2) Decompensation orthodontic treatment for orthognathic surgery: indicated for individuals with occlusal scores Goslon 4 and 5 or patients with Goslon 3 coupled with an unpleasant facial esthetics. In nearly $30 \%$ of individuals, orthognathic surgery will be necessary for treatment finalization. It should be mentioned that the maxillary incisors do not require decompensation movements during preoperative orthodontic treatment, because individuals with clefts and Class III facial pattern present normal inclination of maxillary incisors due to the restrictive effect of the operated upper lip. Mandibular incisors may be proclined in the presence of a wide symphisis and good quality of gingiva, in patients with mild dental crowding. Orthognatic surgery is performed after the completion of facial growth and usually involves Le Fort I osteotomy for maxillary advancement.

\subsection{3-FINALIZATION AND RETENTION}

The finalization of orthodontic treatment of individuals with clefts follows the same principles of orthodontic treatment in individuals without clefts. The dental arches should have been coordinated throughout orthodontic movement, and adequate intercuspation should be achieved, with positive overjet and overbite. The orthodontist should always check the functional occlusion of the individual, to achieve occlusal comfort and the longevity of dental and periodontal tissues. Due to 

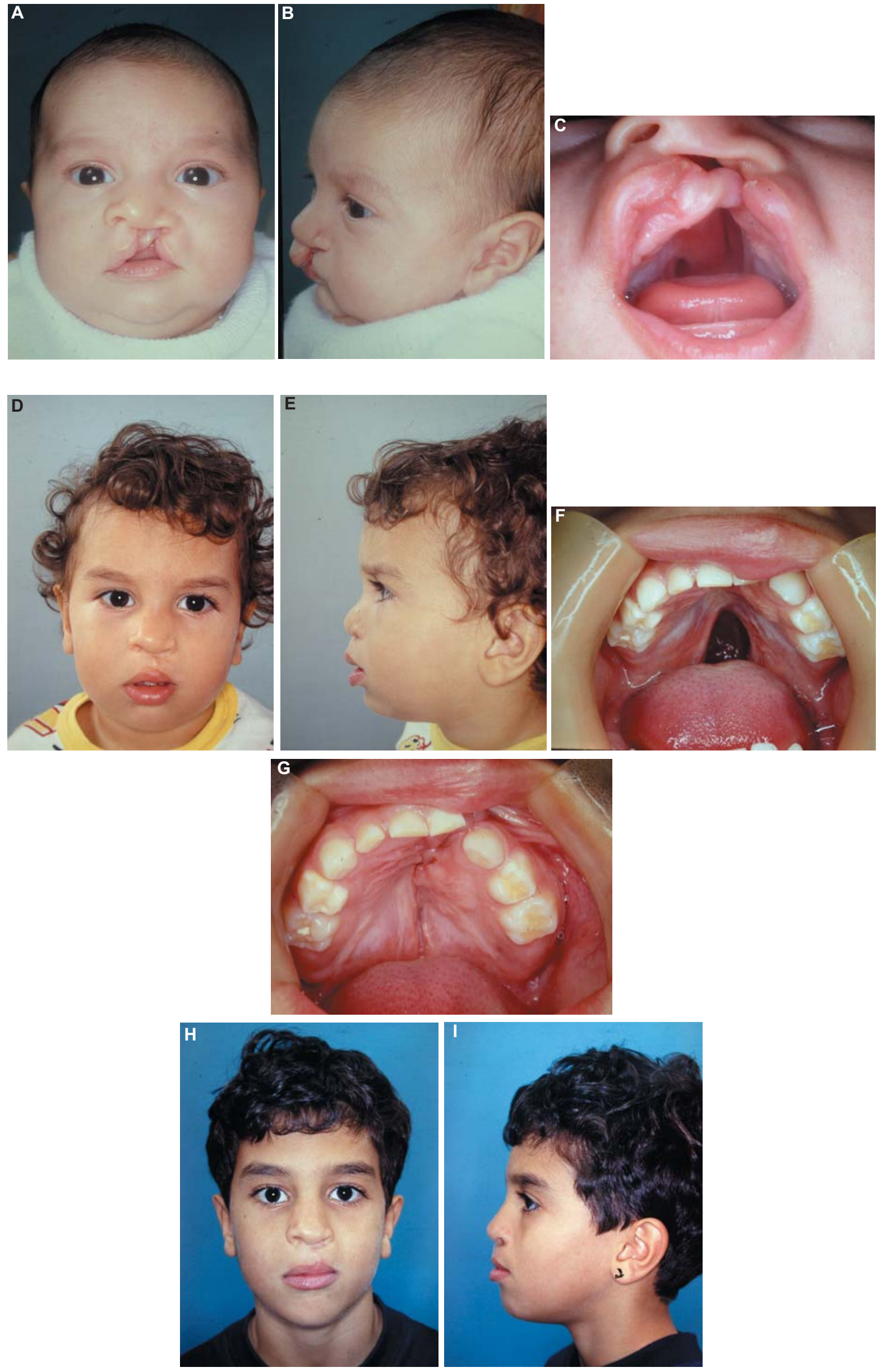

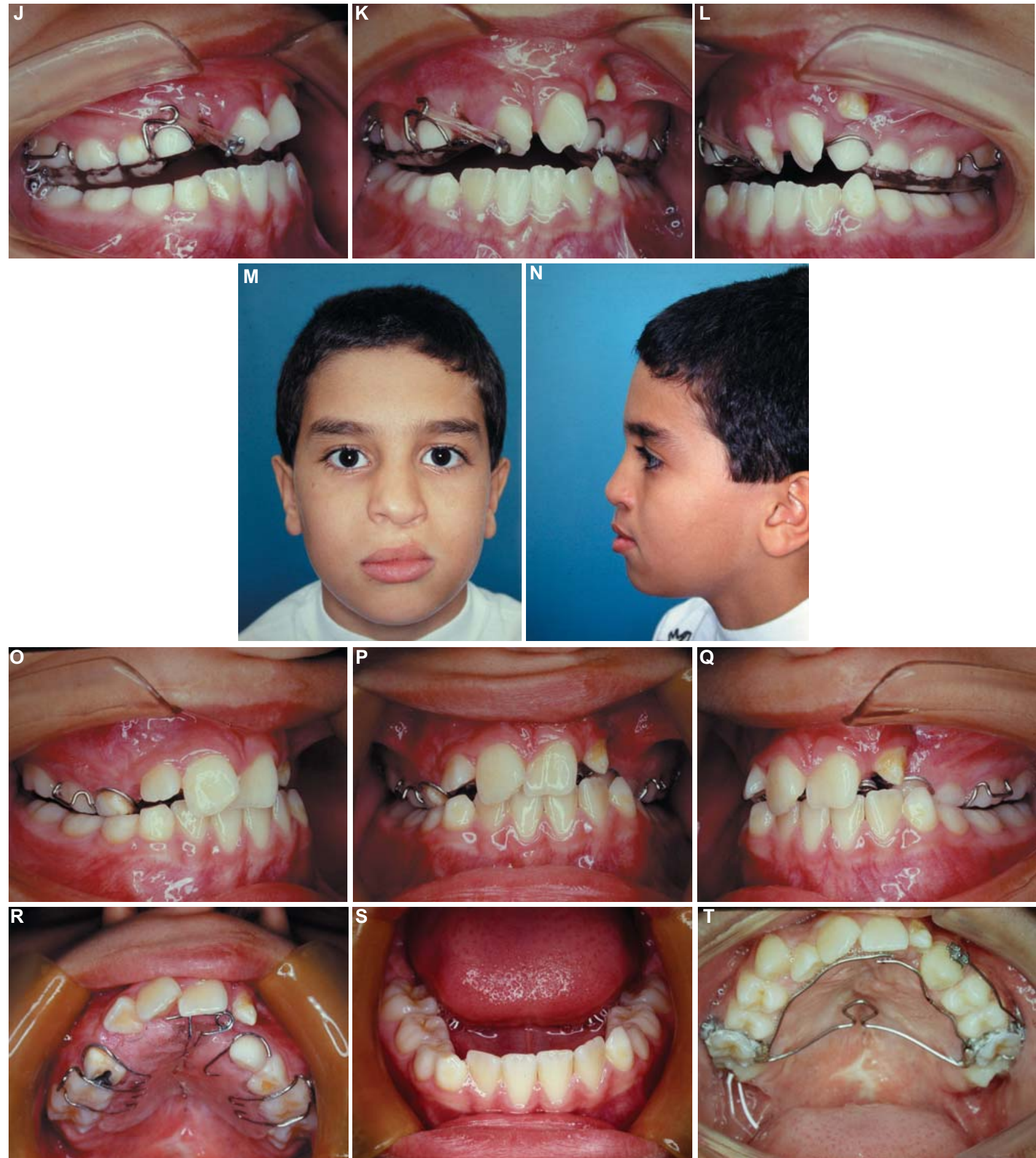

Figure 4- Left unilateral complete cleft lip and palate case. 4A to 4C- before lip and palate repair. 4D to 4F- after lip repair. 4G- after palate repair. 4H to 4S- beginning of orthodontic treatment and dental irruption control. 4T- after rapid maxillary expansion with Haas type expander as described in Figure 3 (parents authorized the publication of these pictures) 
FREITAS JAS, GARIB DG, OLIVEIRA TM, LAURIS RCMC, ALMEIDA ALPF, NEVES LT, TRINDADE-SUEDAN IK, YAEDÚ RYF, SOARES S, PINTO JHN
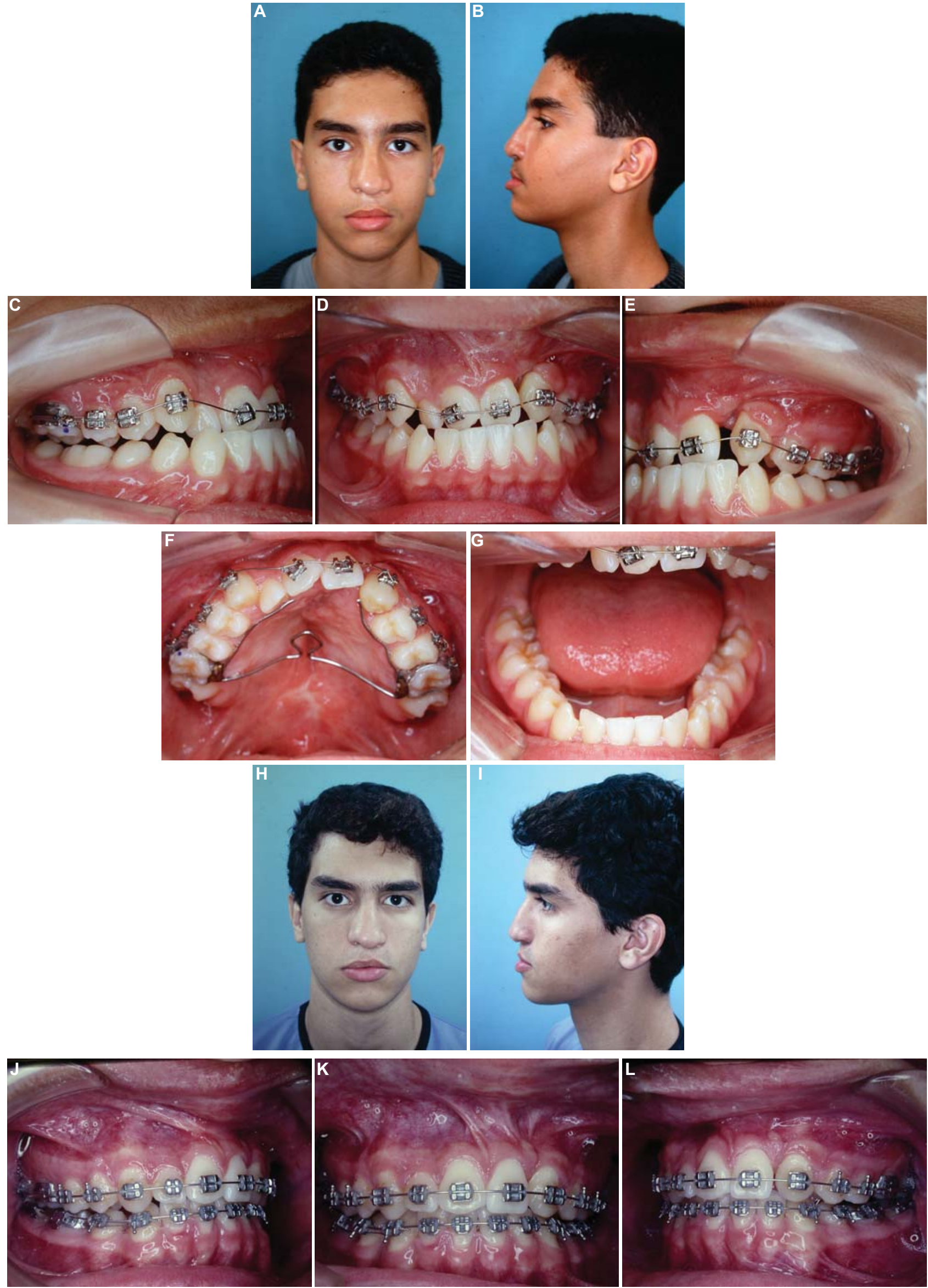

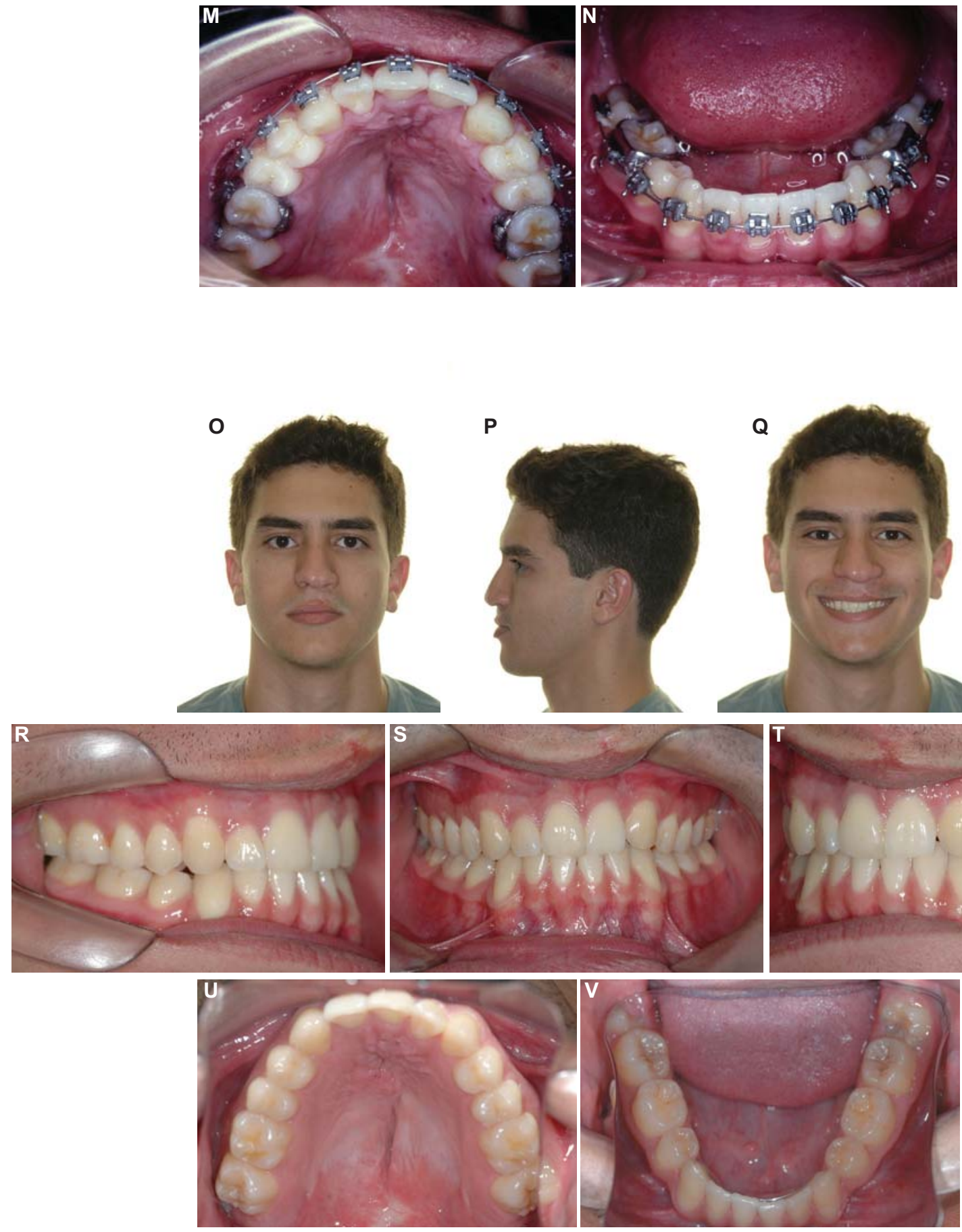

Figure 5- Continuation of the case of figure 4 after secondary bone graft procedure. $5 \mathrm{~A}$ to $5 \mathrm{~N}$ - fixed appliance with uncrossing bite mechanics and use of the canine as lateral incisor. 50 to $5 \mathrm{~V}$ - after fixed appliance removal (parents authorized the publication of these pictures) 
the frequent aforementioned hypodontia, the molar relationship is often finalized in Class II relationship at the cleft side. Thus, the laterality should be adjusted for group disocclusion.

After all steps of orthodontic treatment, the retention period is initiated, which is very important in the rehabilitation of individuals with clefts. The disrupted maxilla is more susceptible to relapse. The bone graft has exclusive action on the alveolar region, leaving the entire cleft palate covered only with soft tissue.

The retention protocol of the Hospital for Rehabilitation of Craniofacial Anomalies - USP involves a modified Hawley appliance in the maxillary arch and $3 \times 3$ retainer in the mandibular arch. The plate often includes prosthetic teeth in the prosthetic spaces that will be rehabilitated later with dental implants or prostheses. Individuals are instructed to wear the Hawley plate continuously in the first year. After one year, if the occlusion is stable, the Hawley plate is used at nighttime for additional one year. After this period, intercalated use is indicated for further 6 months until definitive discontinuity of use. The mandibular $3 \times 3$ retainer is maintained permanently in patients with a good level of oral hygiene. However, regular professional prophylaxis is recommended. Patients are followed for 3 to 5 years after the appliance removal. At this period, the orthodontist should also follow the third molar eruption, with referral for extraction in opportune timing.

\section{FINAL CONSIDERATIONS}

The pediatric dentistry follows patients from growth to adolescence with the main goal of motivate breastfeeding and maintain a high level of oral hygiene. Additionally, the pediatric dentistry team must diagnose the malocclusions and refer for orthodontic treatment in adequate timing.

Orthodontic diagnosis is based on conventional orthodontic records, as well as the valuable occlusal scores. The protocol of orthodontic interventions should be simplified and consistent, prioritizing the approaches that have a significant impact on the final outcome. Very early orthodontic interventions should be avoided because they present poor stability and make the rehabilitation even more exhaustive for the individuals and their families. Orthodontic treatment planning should consider the particular expectations of the individual, as well as the wide individual variability of morphological characteristics of the face and occlusion.

\section{REFERENCES}

1- Alves KM, Peixoto V, Gomide MR, Carvalho Carrara CF, Costa B. Prevalence of palatal and alveolar cysts in babies with cleft lip and palate. Cleft Palate Craniofac J. 2004;41(5):490-3.

2- Atack N, Hathorn I, Mars M, Sandy J. Study models of 5 year old children as predictors of surgical outcome in unilateral cleft lip and palate. Eur J Orthod. 1997;19:165-70.

3- Bartzela T, Katsaros C, Shaw WC, Rønning E, Rizell S, Bronkhorst $E$, et al. A longitudinal three-center study of dental arch relationship in patients with bilateral cleft lip and palate. Cleft Palate Craniofac J. 2010;47(2):167-74.

4- Bergland O, Semb G, Abyholm FE. Elimination of the residual alveolar cleft by secondary bone grafting and subsequent orthodontic treatment. Cleft Palate J. 1986;23(3):175-205.

5- Bessell A, Hooper L, Shaw WC, Reilly S, Reid J, Glenny AM. Feeding interventions for growth and development in infants with cleft lip, cleft palate or cleft lip and palate. Cochrane Database Syst Rev. 2011 Feb 16;(2):CD003315.

6- Britton KF, Welbury RR. Dental caries prevalence in children with cleft lip/palate aged between 6 months and 6 years in the West of Scotland. Eur Arch Paediatr Dent. 2010;11(5):236-41.

7- Cabete HF, Gomide MR, Costa B. Evaluation of primary dentition in cleft lip and palate children with and without natal/neonatal teeth. Cleft Palate Craniofac J. 2000;37(4):406-9.

8- Capelozza Filho L, Normando AD, Silva Filho OG. Isolated influences of lip and palate surgery on facial growth: comparison of operated and unoperated male adults with UCPL. Cleft PalateCraniofac J. 1996;33(1):51-6.

9- Carvalho Carrara CF, Oliveira Lima JE, Carrara CE, Gonzalez Vono B. Chronology and sequence of eruption of the permanent teeth in patients with complete unilateral cleft lip and palate. Cleft Palate Craniofac J. 2004;41(6):642-5.

10- Castilho AR, Neves LT, Carvalho Carrara CF. Evaluation of oral health knowledge and oral health status in mothers and their children with cleft lip and palate. Cleft Palate Craniofac J. 2006;43(6): 726-30.

11- Cheng LL, Moor SL, Ho CT. Predisposing factors to dental caries in children with cleft lip and palate: a review and strategies for early prevention. Cleft Palate Craniofac J. 2007;44(1):67-72. 12- Chiu Y, Liao Y, Chen PK. Initial cleft severity and maxillary growth in patients with complete unilateral cleft lip and palate. Am J Orthod Dentofac Orthop. 2011;140(2):189-95.

13- Dalben GS, Gomide MR, Costa B, Neves LT. Description of a clinical technique for tooth extraction in the cleft lip and palate area. Int J Paediatr Dent. 2001;11(2):143-6.

14- Duque C, Dalben GS, Aranha AM, Carrara CF, Gomide MR, Costa B. Chronology of deciduous teeth eruption in children with cleft lip and palate. Cleft Palate Craniofac J. 2004;41(3):285-9. 15- Freitas JA, Neves LT, Almeida AL, Garib DG, Trindade-Suedam IK, Yaedú RY, et al. Rehabilitative treatment of cleft lip and palate: experience of the Hospital for Rehabilitation of Craniofacial Anomalies/USP (HRAC/USP) - Part I: overall aspects. J Appl Oral Sci. $2012 ; 20(1): 9-15$.

16- Galante JM, Costa B, Carvalho Carrara CF, Gomide MR. Prevalence of enamel hypoplasia in deciduous canines of patients with complete cleft lip and palate. Cleft Palate Craniofac J. 2005;42(6):675-8.

17- Garib DG, Yatabe MS, Ozawa TO, Silva Filho OG. Alveolar bone morphology in patients with bilateral complete cleft lip and palate in the mixed dentition: cone beam computed tomography evaluation. Cleft Palate Craniofac J. 2012;49(2):208-14.

18- Gomes AC, Neves LT, Gomide MR. Enamel defects in maxillary central incisors of infants with unilateral cleft lip. Cleft Palate Craniofac J. 2009;46(4):420-4.

19- Kobayashi TY, Gomide MR, Carrara CF. Timing and sequence of primary tooth eruption in children with cleft lip and palate. J Appl Oral Sci. 2010;18(3):220-4. 
20- Letra A, Menezes R, Granjeiro JM, Vieira AR. Defining subphenotypes for oral clefts based on dental development. ] Dent Res. 2007;86(10):986-91.

21- Liao YF, Mars M. Hard palate repair timing and facial growth in cleft lip and palate: a systematic review. Cleft Palate Craniofac J. 2006;43:563-70.

22- Liao YF, Mars M. Hard palate repair timing and facial morphology in unilateral cleft lip and palate: before versus after pubertal peak velocity age. Cleft Palate Craniofac J. 2006;43(3):259-65.

23- Maciel SP, Costa B, Gomide MR. Difference in the prevalence of enamel alterations affecting central incisors of children with complete unilateral cleft lip and palate. Cleft Palate Craniofac J. 2005;42(4):392-5.

24- Mars M, Plint DA, Houston WJ, Bergland O, Semb G. The Goslon Yardstick: a new system of assessing dental arch relationships in children with unilateral clefts of the lip and palate. Cleft Palate $\mathrm{J}$. $1987 ; 24(4): 314-22$.

25- Medeiros AS, Gomide MR, Costa B, Carrara CF, Neves LT. Prevalence of intranasal ectopic teeth in children with complete unilateral and bilateral cleft lip and palate. Cleft Palate Craniofac J. $2000 ; 37(3): 271-3$.

26- Menezes R, Vieira A. Dental anomalies as part of the cleft spectrum. Cleft Palate Craniofac J. 2008;45(4):414-9.

27- Mølsted K, Brattström V, Prahl-Andersen B, Shaw WC, Semb G. The Eurocleft study: intercenter study of treatment outcome in patients with complete cleft lip and palate. Part 3: dental arch relationships. Cleft Palate Craniofac J. 2005;42(1):78-82.

28- Mossey PA. The heritability of malocclusion: Part 1 - Genetics, principles and terminology. $\mathrm{Br}$ J Orthod. 1999;26(2):103-13.

29- Ozawa TO, Shaw WC, Katsaros C, Kuijpers-Jagtman AM, Hagberg C, Rønning E, et al. A new yardstick for rating dental arch relationship in patients with complete bilateral cleft lip and palate. Cleft Palate Craniofac J. 2011;48(2):167-72.

30- Ramos MM, Carrara CF, Gomide MR. Parental acceptance of behavior management techniques for children with clefts. J Dent Child (Chic). 2005;72(2):74-7.

31- Ribeiro LL, Neves LT, Costa B, Gomide MR. Dental anomalies of the permanent lateral incisors and prevalence of hypodontia outside the cleft area in complete unilateral cleft lip and palate. Cleft Palate Craniofac J. 2003;40(2):172-5.

32- Ribeiro LL, Neves LT, Costa B, Gomide MR. Dental development of permanent lateral incisor in complete unilateral cleft lip and palate. Cleft Palate Craniofac J. 2002;39(2):193-6.

33- Semb G. A study of facial growth in patients with bilateral cleft lip and palate treated by the Oslo CLP Team. Cleft Palate Craniofac J. 1991;28(1):22-39; discussion 46-8.
34- Semb G. A study of facial growth in patients with unilateral cleft lip and palate treated by the Oslo CLP Team. Cleft Palate Craniofac J. 1991;28(1):1-21; discussion 46-28.

35- Semb G, Brattström V, Mølsted K, Prahl-Andersen B, Shaw WC. The Eurocleft study: intercenter study of treatment outcome in patients with complete cleft lip and palate. Part 1: introduction and treatment experience. Cleft Palate Craniofac J. 2005;42:64-8. 36- Semb G, Shaw WC. Simonart's band and facial growth in unilateral clefts of the lip and palate. Cleft Palate Craniofac J. 1991;28:40-6; discussion 46-8

37- Silva AP, Costa B, Carvalho Carrara CF. Dental anomalies of number in the permanent dentition of patients with bilateral cleft lip: radiographic study. Cleft Palate Craniofac J. 2008;45(5):473-6. 38- Silva Dalben G, Costa B, Gomide MR, Neves LT. Dental anesthetic procedures for cleft lip and palate patients. J Clin Pediatr Dent. $2000 ; 24(3): 153-8$.

39- Silva Dalben G, Costa B, Gomide MR, Teixeira das Neves LT. Breast-feeding and sugar intake in babies with cleft lip and palate. Cleft Palate Craniofac J. 2003;40(1):84-7.

40- Silva Filho OG, Albuquerque MV, Kurol J. Ectopic eruption of maxillary first permanent molars in children with cleft lip. Angle Orthod. 1996;66(5):373-80.

41- Silva Filho OG, Castro Machado FM, Andrade AC, Souza Freitas JA, Bishara SE. Upper dental arch morphology of adult unoperated complete bilateral cleft lip and palate. Am J Orthod Dentofacial Orthop. 1998;114(2):154-61.

42- Silva Filho OG, Normando AD, Capelozza Filho L. Mandibular growth in patients with cleft lip and/or cleft palate - the influence of cleft type. Am J Orthod Dentofacial Orthop. 1993;104:269-75. 43- Silva Filho OG, Ramos AL, Abdo RC. The influence of unilateral cleft lip and palate on maxillary dental arch morphology. Angle Orthod. 1992;62(4):283-90.

44- Silva Filho OG, Rosa LA, Lauris RC. Influence of isolated cleft palate and palatoplasty on the face. J Appl Oral Sci. 2007;15:199208.

45- Tereza GP, Carrara CF, Costa B. Tooth abnormalities of number and position in the permanent dentition of patients with complete bilateral cleft lip and palate. Cleft Palate Craniofac J. 2010;47(3):247-52.

46- Trindade IEK, Silva Filho OG. Fissuras labiopalatinas - Uma abordagem interdisciplinar. Livraria Santos Editora Ltda 2007;1:1337.

47- World Health Organization. Global strategies to reduce the heath-care burden of craniofacial anomalies. Geneva: The Organization; 2002. 